\title{
KAWASAKI DISEASE WITH EVOLUTION OF GIANT ANEURYSMS IN THE ACUTE PHASE
}

Marne Rodrigues Pereira Almeida ${ }^{1, *}$, Aline Garcia Islabão ${ }^{1}$, Cristina Medeiros Ribeiro de Magalhães ${ }^{1}$, Maria Custódia Machado Ribeiro ${ }^{1}$ Luciano Junqueira Guimarães ${ }^{2}$, Agatha Siqueira Afonso ${ }^{1}$, Caio Alexandre Zanoni ${ }^{1}$, Marlon Sousa Lopes ${ }^{1}$, Regiane Minardi Duque Neves ${ }^{1}$, Sarah Polyane Veloso ${ }^{1}$

1.Hospital da Criança de Brasília José de Alencar, Brasília (DF), Brazil. 2.Instituto Hospital de Base do Distrito Federal, Brasília (DF), Brazil.

*Corresponding author: marnerp@gmail.com

\section{BACKGROUND}

Kawasaki disease (KD) is an acute, febrile vasculitis mainly affecting children younger than 5 years of age. This disease often involves medium-sized arteries, especially coronary arteries. Circa 15-25\% of untreated children will develop coronary artery abnormalities (CAAs) including coronary dilatation and aneurysms. Coronary artery abnormalities resulted from KD constitutes an important cause of acquired heart disease in children. Fatality rate decreased markedly after the introduction of intravenous immunoglobulin (IVIG) therapy in the late 1980s. Timely initiation of treatment with IVIG reduces the incidence of CAAs from 25 to $4 \%$. Cardiovascular sequelae of Kawasaki disease may include threatening conditions like artery aneurysms and aneurysm with thrombosis, mainly in coronary arteries. The giant coronary's enlargement occurring since the acute phase of Kawasaki disease is an uncommon complication and the outcome of interventional approaches still poorly studied. Prompt diagnosis is essential and an updated algorithm defines supplemental information to be used to assist the diagnosis when classic clinical criteria are incomplete. Although intravenous immunoglobulin is the main initial treatment, the role for additional primary therapy in selected patients is considered. Approximately 10 to $20 \%$ of patients do not respond to initial intravenous immunoglobulin, and recommendations for additional therapies are provided. Careful initial management of evolving coronary artery abnormalities is essential, necessitating an increased frequency of assessments and escalation of thromboprophylaxis. Risk stratification for long-term management is based primarily on maximal coronary artery luminal dimensions, normalized as Z-scores, and is calibrated to both past and current involvement. Patients with aneurysms require life-long and uninterrupted cardiology follow-up.

\section{CASE REPORT}

B.B.A., male patient, 9 months of age, was previously healthy. He was admitted with fever for 12 days, 3 to 4 daily episodes, unresponsive to the administration of antipyretics, associated with urticarial rash on trunk and in the upper and lower limbs started on the first day of fever and still lasting. Additionally, there was reactivation of the BCG vaccine scar. On the third day of the fever, edema and hyperemia were noted in the extremities, which subsequently developed laminar periungual desquamation. On the seventh day of evolution, Kawasaki's disease was suspected, in its incomplete form. Cardiac involvement was identified by coronary aneurysms (December 2019): on the right distal coronary (Z-score +4); at left coronary (Z-score of +6.5) and anterior descending artery (Z-score +3). An immunoglobulin dose $(2 \mathrm{~g} / \mathrm{kg}$ ) was prompted prescribed and, $72 \mathrm{~h}$ after, the fever seemed to have ceased. However, on the 15th day of evolution, fever had returned in a similar pattern, so the use of human immunoglobulin was repeated at the same dosage, this time associated with methylprednisolone. At first, the fever subsided with the administration of this last regimen, but after 3 days, there was a fever and skin lesions upsurge. This was on the 19th day of disease evolution. The use of systemic corticosteroid continued, now associated with the use of acetylsalicylic acid (ASA) in an antiplatelet aggregation regimen. The child remained with fever, but had an involution of the skin signs. Based on a treatment flow chart proposed in 2017 by the American Heart Association, infliximab was prescribed ( $5 \mathrm{mg} / \mathrm{kg} / \mathrm{dose}$ ). Despite the infliximab use, the patient still remained febrile for $48 \mathrm{~h}$ after the infusion, so cyclosporine was opted for. At this time, an infectious disease screening was done, and prophylactic ceftriaxone was used. The patient became afebrile for about $48 \mathrm{~h}$ after the start of immunosuppressant therapy. The sequential echocardiography control showed an aneurysmatic increase (January 2020) at left coronary trunk (Z-score +8.89); right coronary artery (Z-score +19.1 ); anterior descending (Z-score $+6.5 \mathrm{~mm}$ ) and pericardial with laminar effusion. Anticoagulation with heparin and beta-blockers were started. Currently, aneurysms are in continuous echocardiographic involution/stabilization findings (September 2020): at left coronary trunk (Z-score +2.4 ); right coronary artery remains with a saccular aneurysm (Z-score +8.21 ); anterior descending with dilated trunk (Z-score $+3.3 \mathrm{~mm}$ ). The use of cyclosporine remained until 06/05/2020. The use of systemic 
corticosteroids remained until April 2020. Currently, the patient is in use of enoxaparin, propranolol and ASA. He has not developed any signs of ischemic cardiac disease until now.

\section{CONCLUSION}

Clinical decision making should be individualized to specific patient circumstances, like when there was a precocious large/giant artery aneurism in a Kawasaki's disease patient. In the present case, with an intensely unresponsive clinical evolution, however, there was clinical stabilization after the successive therapeutic staggerings. Giant aneurysms are a relative uncommon complication of KD but can lead to significant sequelae and poor outcome. This complication is characterized as hyperinflammation in serum and is more prone to developing IVIG nonresponsiveness and CAAs and aseptic meningitis. Increased production of cytokines as IL-6, IL-10, TNF- and IFN- may pay a key role in the pathogenesis of aneurysms. The paired cytokine studies may be helpful to define the cytokines' role in KD, but this analysis is not possible. 